\begin{tabular}{|c|l|}
\hline Title & Gel friction: A model based on surface repul sion and adsorption \\
\hline Author(s) & Gong, Jianping; Osada, Y oshihito \\
\hline Citation & $\begin{array}{l}\text { The Journal of Chemical Physics, 109(18), 8062-8068 } \\
\text { https://doi.org/10.1063/1.477453 }\end{array}$ \\
\hline Issue Date & 1998-11-08 \\
\hline Doc URL & http://hdl.handle.net/2115/5721 \\
\hline Rights & Copyright $\odot$ 1998 A merican Institute of Physics \\
\hline Type & article \\
\hline File Information & JCP109-18.pdf \\
\hline
\end{tabular}

Instructions for use 


\title{
Gel friction: A model based on surface repulsion and adsorption
}

\author{
Jianping Gong and Yoshihito Osada \\ Division of Biological Sciences, Graduate School of Science, Hokkaido University, Sapporo 060-0810, \\ Japan
}

(Received 1 June 1998; accepted 4 August 1998)

\begin{abstract}
A model describing the frictional force produced when a polymer gel is sliding on a solid surface has been proposed from the viewpoint of solvated polymer repulsion and adsorption theory at a solid surface. General relations for the frictional force $f$ expressed as functions of the normal loading $P$, sliding velocity $\nu$, the polymer volume fraction $\phi$, or the elastic modulus $E$ of the gel, etc., have been derived by applying scaling relations to the model. For the repulsive case, $f$ is ascribed to the viscous flow of solvent at the interface and $f$ is theoretically demonstrated to be proportional to the sliding velocity $\nu$ and the normal pressure $P$ when the pressure is smaller than the elastic modulus of the gel. For the attractive case, in addition to the hydrodynamic friction, the force to detach the adsorbing chain from the substrate appears as friction. When $\nu$ is not very large, $f \propto \nu$. At an intermediate velocity, $f$ has a velocity dependence less than linear, depending on the strength of adsorption. At a higher sliding velocity, $f \propto \nu$ again since the hydrodynamic friction becomes predominant at this stage. The theoretical results coincide well with some experimental observations that confirm the essential feature of the model. (c) 1998 American Institute of Physics.
\end{abstract}

[S0021-9606(98)70342-3]

\section{INTRODUCTION}

A gel consists of an elastic cross-linked macromolecular network with a liquid filling the interstitial space of the network. The network holds the liquid in place through its interaction forces and so gives the gel solidity and coherence, but the gel is also wet and soft and capable of undergoing large deformation.

In a previous short letter, ${ }^{1}$ we reported the specific frictional behavior of various kinds of gels sliding on a solid surface. The frictional force of a gel shows complicated dependencies on the load $W$, the apparent contact area A of two surfaces, and on the sliding velocity $\nu$. Additionally, $\mu$ changes in a wide range and reaches a value as low as $10^{-3}$, which has only been observed in some biological systems, such as in the human and animal joints. Animal cartilage, which consists of a three-dimensional collagen network filled with a synovial fluid, has a friction coefficient as low as $10^{-3}$. $^{2}$

Apparently, the mechanism of the frictional force produced by sliding a gel against a solid surface is quite different from that produced by two solid surfaces sliding over each other. According to Amonton's law, the frictional force $F$ between two solids is proportional to the load $W$ forcing them together $F=\mu W .^{3}$ The proportional coefficient $\mu$, known as the frictional coefficient, depends on neither the sliding speed nor the apparent contact area of two surfaces but only depends on the moving materials. $\mu$ usually lies in a range of $0.5-1.0 .^{4}$

The aim of this paper is to derive a general equation describing the frictional behaviors of the water-swollen gel that slides on a solid surface. We propose a model from the viewpoint of polymer-solid surface repulsion and adsorption to describe the frictional behavior of a gel sliding on a solid surface. When a polymer solution is placed in contact with a solid wall, the polymer chain would be either repelled from or adsorbed on the solid wall depending on the strength of the interaction between them in comparison with the solvent. The behavior of a cross-linked gel surface is, from a certain point of view, similar to that of a polymer solution when being contacted with a solid wall. The polymer chains on the surface of the gel will be repelled from the surface if it is repulsive, or will be adsorbed on the solid surface if it is attractive. In the former case, the viscous flow of solvent between the solid surface and the polymer will make a dominant contribution to the frictional force. In the latter case, however, the adsorbing chain will be stretched when the solid surface is in motion relative to the gel. The elastic force increases with the deformation and detaches the adsorbing polymer chain from the substrate, thus it will in turn appear as the frictional force.

\section{MODEL}

On the bases of these considerations, we have tried to deduce equations of the frictional force expressed as functions of experimental parameters such as the load, velocity, volume fraction of the polymer, or the elastic modulus of the gel. In order to simplify the problem, we confine ourselves to following conditions.

(1) The system is immersed in a solvent. The solvent is athermal to the polymer, which means the excluded volume parameter equals the cubic of the monomer length. This confinement permits us to use many scaling results.

(2) The interface between the gel and the solid has a contact in a macromolecular level.

(3) The gel has the same surface repulsion or adsorption as 
that of a linear polymer solution, neglecting the crosslinking effect of the gel on the interface interaction.

(4) The interfacial motion does not involve any chain scission, that is, motion occurs by desorption in the attractive case.

Scaling theory describes a gel as a collection of adjacent blobs with a radius $R_{F}$, each blob being associated with one partial polymer chain and having a conformation similar to that of a single linear chain. $R_{F}$ is equivalent to the radius of a flexible polymer chain in an athermal solvent in consideration of the volume excluded effect ${ }^{5}$

$$
R_{F} \cong a N^{3 / 5} \text {. }
$$

Here, $a$ is the effective length of the monomer and $N$ is the degree of polymerization. For a gel, $N$ is the number of monomer units between two cross-linking points. Therefore, we can treat a partial chain of a gel in a similar way to that of a solvated polymer and use scaling results on the polymersolid interface to deduce gel friction.

\section{A. Repulsive substrate}

We begin with the scaling result for the interaction between a polymer solution and a solid wall. When the interaction of the monomeric unit of the polymer with the solid surface is weaker than that of the solvent, the polymer would be repelled from the interface and a surface depleted layer forms. According to scaling relations, this depleted layer has a thickness equivalent to the correlation length $\xi$ for a semidilute linear polymer solution, ${ }^{5}$

$$
\xi \cong a \phi^{-3 / 4},
$$

where $\phi$ is the volume fraction of polymer. The interface energy between the polymer solution and the solid surface $A$ is $^{5}$

$$
A-A_{0} \cong \Pi_{0} \xi,
$$

where $A_{0}$ is the interface energy between the substrate and the pure solvent and $\Pi_{0}$ is the osmotic pressure of the bulk solution that is given by ${ }^{5}$

$$
\Pi_{0} \cong T \xi^{-3} \text {. }
$$

Equation (3) means that the work done by the solid surface to repel the polymer from the surface against the osmotic pressure should be equal to the increase in the surface energy. This surface repulsion also occurs for a gel. Since a gel is cross linked, its total osmotic pressure is zero. This means that when a gel is put on a repulsive substrate, it will be repelled to an infinite distance if the weight of the gel is negligibly small. Since the interface energy between the gel and the substrate should be the same as that of a polymer solution with a corresponding concentration, the repelled distance $\xi_{g}$ of the gel from the solid surface against a normal pressure $P$ can be determined by

$$
P \xi_{g} \cong A-A_{0} \cong \Pi_{0} \xi \text {. }
$$

Accordingly, no surface adsorption of the polymer occurs, and the frictional force arises from the viscous flow of the solvent at the interface.
Since the viscous flow of solvent obeys Newton's law, we can apply the hydrodynamic lubrication between two parallel plates filled with solvent to obtain the friction force, ${ }^{6}$

$$
f=\frac{\eta \nu}{\xi_{g}+D}
$$

Here, two no-slip boundaries have been used. One is, needless to say, on the solid surface; the other is in the gel, on a plane parallel to the interface, of one partial chain radius of $D$ depth from the gel surface. $D$ can be determined as follows.

When a polymer chain with an unperturbed radius $R_{F}$ is compressed to a thickness $D$ in the direction parallel to the pressure, it will be stretched to a length $R_{\|}$in the direction vertical to the pressure. The relation between $D$ and $R_{\|}$is given by ${ }^{5}$

$$
R_{\|} \cong N a\left(\frac{a}{D}\right)^{2 / 3} .
$$

The free energy of the polymer chain under this state is ${ }^{5}$

$$
F \cong T N\left(\frac{a}{D}\right)^{5 / 3}
$$

At equilibrium,

$$
\left(\frac{\partial F}{\partial V}\right)_{N}=-\left(P+\Pi_{0}\right),
$$

where $V=D^{2} R_{\|}$is the volume of the polymer chain. From Eq. (9), we get the relation

$$
D^{3}=T /\left(P+\Pi_{0}\right) \text {. }
$$

This indicates that the thickness of the layer $D$ would decrease under a normal pressure $P$. Since $\Pi_{0} \cong T \xi^{-3}$ and $\xi=R_{F}$ for a gel, we have $D=R_{F} \cong a \phi^{-3 / 4}$ when $P=0$. This result corresponds to the case of no deformation in the absence of external pressure. Since the elastic modulus of a gel, ${ }^{5}$

$$
E \cong c T / N,
$$

where

$$
c=\phi / a^{3}
$$

is the polymer concentration, $N=\phi^{-5 / 4}$ for a gel, we have

$$
E \cong T \phi^{9 / 4} / a^{3}=T R_{F}^{-3} \cong \Pi_{0} .
$$

That is, the elastic modulus of a gel is equal to the osmotic pressure of the corresponding linear polymer solution. Therefore, we can express Eq. (6) in terms of $E$ :

$$
f \cong \frac{\eta \nu P}{E^{2 / 3} T^{1 / 3}} \frac{1}{1+(P / E) /(1+P / E)^{1 / 3}} .
$$

When $P / E \ll 1$,

$$
f \cong \frac{\eta \nu P}{E^{2 / 3} T^{1 / 3}}
$$

In order to investigate the effect of solvent content on the friction, we rewrite Eq. (15) as a function of polymer volume fraction, 


$$
f \cong \frac{\eta \nu P a^{2}}{T \phi^{3 / 2}}
$$

Accordingly, for a repulsive substrate, the friction is attributed to the viscous flow of the solvent layer between the gel and the substrate. The static friction is zero and $f$ is proportional to the sliding velocity and the normal pressure applied when it is smaller than the elastic modulus of the gel. Equation (16) also indicates that the frictional force will decrease with an increase in the polymer volume fraction. The physical meaning of this inverse relation is that the higher the polymer volume fraction, the stronger the repulsion at the interface and hence the thicker the solvent layer.

\section{B. Attractive substrate}

When the polymer has a stronger interaction with the solid surface than the solvent, adsorption occurs. Let us consider a thermodynamically reversible adsorption. At the equilibrium state, the rate of adsorption and desorption is the same. If we monitor one macromolecule, it adsorbs on the surface for an average lifetime $\tau_{b}$. After $\tau_{b}$, it spontaneously desorbs from the surface. It takes a time $\tau_{f}$ before the polymer adsorbs on the surface again. The relation between $\tau_{b}$ and $\tau_{f}$ is given by

$$
\tau_{b}^{-1}=\tau_{f}^{-1} \exp \left(-F_{\text {ads }} / T\right)
$$

here $F_{\text {ads }}$ is the adsorption energy of one chain. When the solid surface is in relative motion to the gel surface, the adsorbing chain would be stretched. The elastic force $f_{\text {el }}$ of the chain increases with time due to the accumulation of stretching and at a deformation of $r=\nu t,{ }^{5}$

$$
f_{\mathrm{el}} \cong T r / R_{F}^{2}=T \nu t / R_{F}^{2} .
$$

Here, $\nu$ is the sliding velocity and $t$ is the time elapsed after adsorption occurred and thus the average value of $t \leqslant \tau_{b}$. The elastic force will detach the adsorbing chain from the substrate and appears as friction. This friction mechanism is quite similar to what occurred when rubber slides against a hard substrate. Therefore, we can follow the outline of the model proposed by Schallamach for the case of dynamic rubber friction to deduce the friction force of a gel on an attractive substrate. ${ }^{7}$ The dynamic friction per unit area due to adsorption can be expressed as ${ }^{7}$

$$
f=\sum_{i=1}^{m} T \nu t_{i} / R_{F}^{2}=m T \nu \tau_{b} / R_{F}^{2} .
$$

Here, $m$ is the number of adsorbing sites per unit area. Since a gel can be treated as a collection of blobs with radius $R_{F}$, the number of chains per unit area is $1 / R_{F}^{2}$. If we treat one chain as one adsorption site, the possible adsorption site is

$$
m_{0} \cong 1 / R_{F}^{2} \text {. }
$$

The fraction of total sliding time during which a polymer chain is in the adsorbing state is $\tau_{b} /\left(\tau_{b}+\tau_{f}\right)$. This is equivalent to the fraction of adsorption site that is at any given instant in the adsorbing state, so

$$
m=m_{0} \tau_{b} /\left(\tau_{b}+\tau_{f}\right) .
$$

Thus, the friction due to adsorption is

$$
f \cong \frac{T \nu \tau_{f}}{R_{F}^{4}} \frac{\left(\tau_{b} / \tau_{f}\right)^{2}}{\left(\tau_{b} / \tau_{f}+1\right)} .
$$

Following Schallamach's model, the adsorption and desorption are attributed to thermal fluctuation. The stretching of the chain would favor desorption, that is, it would lower the energy difference between the adsorbing state and the free state by a value of $F_{\mathrm{el}}$. Here, $F_{\mathrm{el}}$ is the elastic energy of one stretched chain,

$$
F_{\mathrm{el}}=\frac{3 T r^{2}}{2 R_{F}^{2}}=\frac{3 T \nu^{2} t^{2}}{2 R_{F}^{2}} .
$$

So the transition rate from the adsorbing state to the free state becomes

$$
\rho=\tau_{f}^{-1} \exp \left[-\left(F_{\mathrm{ads}}-F_{\mathrm{el}}\right) / T\right] .
$$

The above relation suggests that the adsorption time $\tau_{b}$ is a function of the deformation rate, or the sliding velocity. Here, instead of a quantity which is linearly proportional to the deformation used in Schallamach's model, the elastic energy of the chain $F_{\text {el }}$, which relates to the deformation in $r^{2}$, is used. So $\rho$ will increase with the deformation more rapidly than in Schallamach's model.

Next, we derive the average lifetimes $\tau_{b}$ and $\tau_{f}$. We first estimate $\tau_{f}$ of a cross-linked polymer chain in the free state. The cross linkage makes it impossible for the polymer chains to change their relative positions, that is, they cannot diffuse independently like a linear chain does. But at a local thermal fluctuation level, the polymer chain of a gel should have a dynamic property similar to that of a linear one. That is, every blob of the gel thermally fluctuates around its equilibrium position like a hard sphere of radius $R_{F}$ does. This consideration permits us to use the Kirkwood approximation to obtain the following relaxation time: ${ }^{5}$

$$
\tau_{f} \cong \eta R_{F}^{3} / T \text {. }
$$

Since the elastic modulus of a gel $E \cong T R_{F}^{-3}$, we have

$$
\tau_{f} \cong \eta / E .
$$

For a typical hydrogel $E=10^{5} \mathrm{~Pa}$ and $\eta=10^{-3} \mathrm{Ns} \mathrm{m}^{-2}$, so $\tau_{f}=10^{-8} \mathrm{~s}$.

$\tau_{b}$ can be obtained following Schallamach's approach,

$$
d m / d t=-\rho m .
$$

By integration of the above equation, we have

$$
m / m_{t=0}=\exp \left[-\int_{0}^{t} \tau_{f}^{-1} u \exp \left(3 \nu^{2} t^{2} / 2 R_{F}^{2}\right) d t\right],
$$

where $u=\exp \left(-F_{\text {ads }} / T\right) . \tau_{b}$ is given by the integral

$$
\tau_{b}=\frac{1}{m_{t=0}} \int_{0}^{\infty} m d t
$$

which can only be solved numerically. When $3 \nu^{2} t^{2} / 2 R_{F}^{2}$ $\ll 1$,

$$
m / m_{t=0}=\exp \left[-\tau_{f}^{-1} u\left(t+\frac{\nu^{2} t^{3}}{2 R_{F}^{2}}\right)\right],
$$




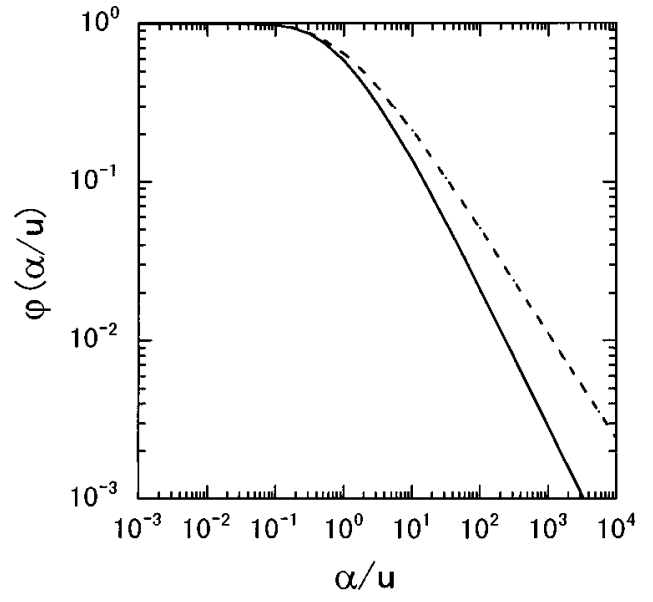

FIG. 1. Normalized average lifetime $u \tau_{b} / \tau_{f}=\varphi(\alpha / u)$ of polymer adsorption as a function of normalized sliding velocity $\alpha / u=\eta \nu / u E^{2 / 3} T^{1 / 3}$. The dotted curve is calculated from the approximation equation of Eq. (31).

$$
u \tau_{b} / \tau_{f}=\int_{0}^{\infty} \exp \left(-x-\frac{\nu^{2} \tau_{f}^{2}}{2 u^{2} R_{F}^{2}} x^{3}\right) d x
$$

The above equation indicates that $u \tau_{b} / \tau_{f}$ is uniquely determined by the parameter $\nu \tau_{f} / u R_{F}$. If we denote $\alpha$ $=\nu \tau_{f} / R_{F}$ and the integration in Eq. (31) as $\varphi(\alpha / u)$,

$$
u \tau_{b} / \tau_{f}=\varphi(\alpha / u) \text {. }
$$

For a certain $u, \tau_{b} / \tau_{f}$ is a function of $\alpha$, which only depends on the sliding velocity. As shown in Fig. 1, when $\alpha / u \rightarrow 0$, $\varphi(\alpha / u) \rightarrow 1$, this corresponds to the equilibrium case of Eq. (17) at $\nu=0$. When $\alpha / u \rightarrow \infty, \varphi(\alpha / u) \rightarrow 0$, this indicates that when the sliding velocity is very large, there would be no adsorption. The dotted line in Fig. 1 is calculated from Eq. (31). When $\alpha / u>0.1$, it begins to deviate from the accurate curve obtained from Eqs. (28) and (29). form,

Using Eq. (32), we can rewrite Eq. (22) in the following

$$
f / E=\frac{\alpha \varphi^{2}(\alpha / u)}{u(u+\varphi(\alpha / u))} .
$$

For a finite $u$, when $\alpha \rightarrow 0, f / E \rightarrow \alpha / u(u+1)$, when $\alpha$ $\rightarrow \infty, f / E \rightarrow 0$. Figure 2 is the relation between $f / E$ and $\alpha$ for fixed $u$ values. The linear relation between $f / E$ and $\alpha$ remains valid when $\alpha / u<0.1$. As can be expected from the results for rubber, $f / E$ has a maximum for a certain $\alpha$. For a certain velocity, $f / E$ increases with an increase in the adsorption energy.

The dimensionless parameter $\alpha=\nu \tau_{f} / R_{F}$ can be expressed in terms of the experimentally measurable parameters by using the result for $\tau_{f}$ :

$$
\alpha=\nu \eta / E^{2 / 3} T^{1 / 3} \text {. }
$$

Thus, the only parameter to be determined in Eq. (33) is $u$ $=\exp \left(-F_{\text {ads }} / T\right)$, which should be affected by the external pressure $P$ applied to the gel. This can be understood by imagining a collection of blobs on a solid surface. These blobs deform and increase their contact with the substrate under the external normal pressure, which suggests that the adsorption energy would increase with the pressure.

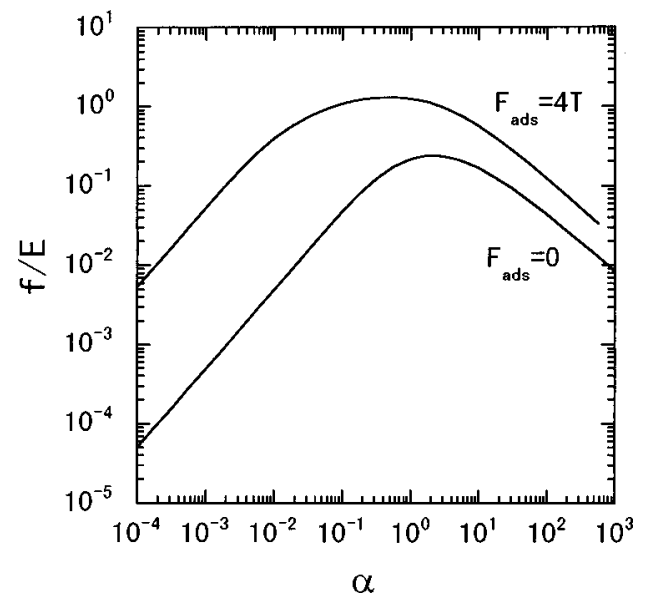

FIG. 2. Normalized frictional force $f / E$ due to adsorption as a function of $\alpha$ for various adsorption energy $F_{\text {ads }}$.

In order to estimate $F_{\text {ads }}$, let us first consider a single polymer chain. When a polymer chain with an unperturbed radius $R_{F}$ is adsorbed on a solid surface, it would deform to a size $D$ in the direction vertical to the surface. The energy due to this deformation is given by Eq. (8) and that due to adsorption is

$$
F_{\mathrm{ads}} \cong T N \delta f_{b} .
$$

Here, $T \delta$ is the effective attraction energy between a monomer unit and the surface in respect to the solvent. $f_{b}$ is the ratio of the partial chain adsorbed, ${ }^{5}$

$$
f_{b} \cong \frac{a}{D} \text {. }
$$

The free energy of the polymer chain in the adsorbing state is $^{5}$

$$
F \cong T N\left(\frac{a}{D}\right)^{5 / 3}-T N \delta f_{b}
$$

We again suppose that these scaling relations are valid for a polymer chain belonging to a network. According to Eq. (36), the adsorption energy is inversely proportional to $D$, we need to discuss the pressure dependence of $D$. At the equilibrium state, Eq. (9) is valid again and we get the relation

$$
\frac{\left(P+\Pi_{0}\right) a^{3}}{T}\left(\frac{D}{a}\right)^{3}+\frac{3 \delta}{5}\left(\frac{D}{a}\right)^{2 / 3}=1 .
$$

By expressing $\Pi_{0}$ in terms of $\phi$, it is found that when $\delta \ll \phi^{1 / 2}$, the second term of Eq. (38) can be neglected and we have Eq. (10) again, which indicates that when the adsorption energy is very small, the deformation is attributed to the external pressure and the deformation due to adsorption can be neglected.

When $\delta \gg \phi^{1 / 2}$, the first term of Eq. (38) can be neglected and we have

$$
D \cong a \delta^{-3 / 2} \text {. }
$$




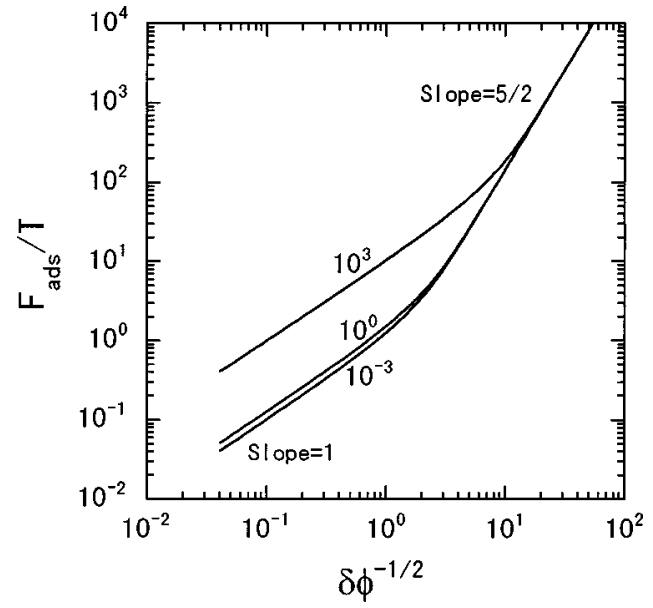

FIG. 3. Dependence of the adsorption energy $F_{\text {ads }}$ of one partial chain on the parameter $\delta \phi^{-1 / 2}$ under various normal pressures. The number in the figure is $P / E$.

This indicates that $D$ is not sensitive to the external pressure when the adsorption is very strong and the deformation is caused by the adsorption. Therefore, we have

$$
F_{\mathrm{ads}} \cong \begin{cases}T \phi^{-1 / 2} \delta\left(1+P a^{3} / \phi^{9 / 4} T\right)^{1 / 3} & \text { for } \delta \ll \phi^{1 / 2} \\ T \phi^{-5 / 4} \delta^{5 / 2} & \text { for } \delta \gg \phi^{1 / 2}\end{cases}
$$

The above relation indicates that for weak adsorption, the adsorption energy of one polymer chain is related to the applied pressure in $F_{\text {ads }} \propto P^{1 / 3}$ when $P$ is relatively larger than the osmotic pressure of the gel since $E \cong T \phi^{9 / 4} / a^{3}$. For strong adsorption, $F_{\text {ads }}$ is not sensitive to the pressure. It should be pointed out that the criteria for weak adsorption is not determined by the condition $\delta \ll 1$ but by $\delta \phi^{-1 / 2} \ll 1$. Figure 3 is the calculated relation between $F_{\text {ads }} / T$ and $\delta \phi^{-1 / 2}$ for various $P / E$ values.

In the case of adsorption, the friction due to the viscous flow of solvent at the vicinity of the interface also exists. By choosing the no-slip boundary at a layer of one blob deep from the gel surface,

$$
f_{\nu} \approx \frac{\eta \nu\left(1-\phi_{s}\right)^{2 / 3}}{D} \text {. }
$$

Here, $\phi_{s}$ is the volume fraction of polymer at the vicinity of solid surface and can be expressed as

$$
\phi_{s}=N f_{b} a^{2} / R_{F}^{2}=\phi^{1 / 4}(a / D) \text {. }
$$

For a highly water swollen gel with a weak adsorption, 1 $-\phi_{s} \approx 1-\phi \approx 1$, we have

$$
f_{\nu} \cong \begin{cases}\eta \nu E^{1 / 3} T^{-1 / 3}(1+P / E)^{1 / 3} & \delta \ll \phi^{1 / 2} \\ \eta \nu \delta^{3 / 2} / a E & \delta \gg \phi^{1 / 2} .\end{cases}
$$

\section{DISCUSSION}

In Sec. II, we have obtained relations for the frictional force of a gel sliding on a solid surface by considering the interaction of the solid surface to the gel surface as repulsive or attractive. For a repulsive substrate, the friction is attributed to the hydrodynamic lubrication of the solvent and Eq. (14) can be rewritten in the following form:

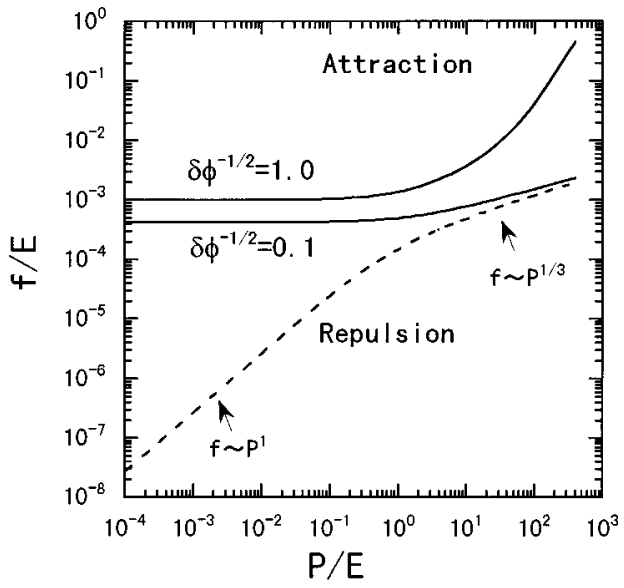

FIG. 4. Pressure dependence of the frictional forces for gels sliding on a repulsive substrate (dotted curve) as well as on attractive substrates with various $\delta \phi^{-1 / 2}$ values (solid curves). $\alpha=2.7 \times 10^{-4}$ was used in the calculation, this value corresponding to $\nu=10^{-4} \mathrm{~m} / \mathrm{s}$ for a gel of $\phi=0.04, a$ $=3 \times 10^{-10} \mathrm{~m}, \eta=10^{-3} \mathrm{Ns} / \mathrm{m}^{2}$, and $T=4.14 \times 10^{-21} \mathrm{~J}(T=300 \mathrm{~K})$.

$$
f_{\text {rep }} / E=\frac{\eta \nu}{E^{2 / 3} T^{1 / 3}} \frac{P / E}{1+(P / E) /(1+P / E)^{1 / 3}} .
$$

For the attractive case, the friction is attributed to two forces: One is the force to detach the adsorbing chain from the substrate. The other is the hydrodynamic friction of solvent. Here, let us further consider weak adsorption that is the case for many highly water swollen hydrogels. For $\delta \phi^{-1 / 2}$ $=0.1$ which satisfies the condition $\delta \ll \phi^{1 / 2}, \quad u \approx 1$ $-\delta \phi^{-1 / 2}(1+P / E)^{1 / 3}$ when $P / E \leqslant 1$. When $\alpha / u$ is small and the approximation $f / E \cong \alpha / u(u+1)$ is valid, the force to detach the chain is

$$
f / E=\frac{\eta \nu}{E^{2 / 3} T^{1 / 3}}\left[\frac{1}{2}+\frac{3}{2} \delta \phi^{-1 / 2}(1+P / E)^{1 / 3}\right] .
$$

The viscous force $f_{\nu}$ of Eq. (43) for weak adsorption can be rewritten as

$$
f_{\nu} / E=\frac{\eta \nu}{E^{2 / 3} T^{1 / 3}}(1+P / E)^{1 / 3} .
$$

So the total friction $f_{\text {attr }}=f+f_{\nu}$ for the attractive substrate of weak adsorption is

$$
f_{\text {attr }} / E=\frac{\eta \nu}{E^{2 / 3} T^{1 / 3}}\left[\frac{1}{2}+\left(1+\frac{3}{2} \delta \phi^{-1 / 2}\right)(1+P / E)^{1 / 3}\right]
$$

at small pressures. By using the relation $E \cong T \phi^{9 / 4} a^{-3}$, Eqs. (44) and (47) can easily be expressed in terms of the polymer volume fraction.

In order to see the magnitude of the friction obtained by the modeling, we calculate the friction values using proper parameters. For the gel, we use the parameters $\phi=0.04, a$ $=3 \times 10^{-10} \mathrm{~m}$, this gives out $E \approx 10^{5} \mathrm{~Pa}$. Other parameters are as follows: $\eta=10^{-3} \mathrm{Ns} / \mathrm{m}^{2}, \quad T=4.14 \times 10^{-21} \mathrm{~J}(T$ $=300 \mathrm{~K}$ ).

Figure 4 shows the calculated curves of the relation between the friction and the normal pressure for a gel sliding on a repulsive substrate (dotted curve) at a velocity $\nu$ 


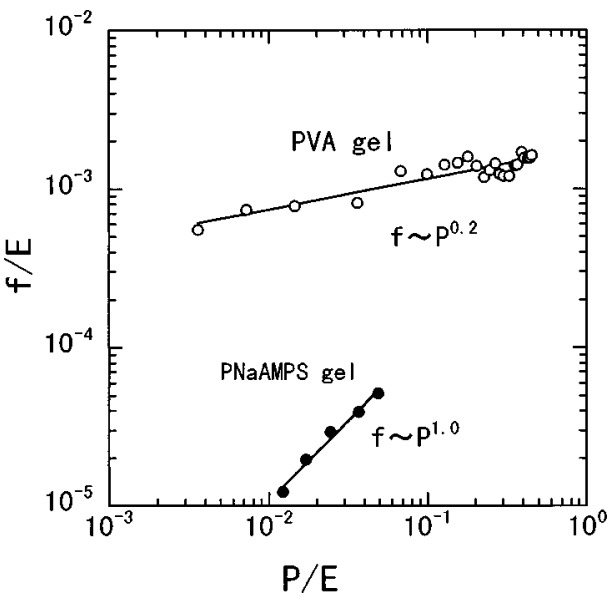

FIG. 5. Experimental results of the dependence of the frictional force $f$ on the pressure $P$ for PVA gel $(\bigcirc)$ and PNaAMPS gel $(\bullet)$ sliding on the glass substrate. $f$ and $P$ are normalized by the elastic modulus of gels $E$. $E=1.5$ $\times 10^{5} \mathrm{~Pa}$ for PVA gel; and $E=1.0 \times 10^{6} \mathrm{~Pa}$ for PNaAMPS gel. Sliding velocity: $7 \mathrm{~mm} / \mathrm{min}$, contact area: $30 \mathrm{~mm} \times 30 \mathrm{~mm}$. Detailed experimental procedures and conditions were described in Ref. 1.

$=10^{-4} \mathrm{~m} / \mathrm{s}$ using Eq. (44). When $P / E \ll 1, f \propto P$, the coefficient of friction for a repulsive substrate $\mu=f / P$ is constant. At a velocity $\nu=10^{-4} \mathrm{~m} / \mathrm{s}, \mu \approx 10^{-3}$. When $P / E \gg 1, f$ $\propto P^{1 / 3}$.

The observed frictional force of the sodium salt of poly(2-acrylamido-2-methylpropanesulfonic acid) (PNaAMPS) gel sliding on a glass substrate showed a linear pressure dependence (Fig. 5). ${ }^{1}$ This is similar to the theoretical prediction. In addition, the magnitude of the friction of the observed data is in the same order with that of the theoretical curve. Besides, the gel showed almost a linear dependence on the sliding velocity. These coincidences suggest that the interaction between the PNaAMPS gel and the glass is repulsive, which is reasonable considering the fact that both the PNaAMPS gel and the glass have negative zeta potentials. ${ }^{8}$

Figure 4 also shows the theoretical curves of the pressure dependence of friction for attractive substrate with various $\delta \phi^{-1 / 2}$ values(solid curves). When $P / E \ll 1$, the friction is not sensitive to pressure. When $P / E \geqslant 1$, the pressure dependence of friction can be divided into three categories: (1) when $\delta \phi^{-1 / 2} \ll 1, f \propto P^{1 / 3}$, as indicated by Eq. (47); (2) when $\delta \phi^{-1 / 2} \approx 1, f$ increases with $P$ rapidly as shown in Fig. 4; (3) when $\delta \phi^{-1 / 2} \gg 1, f \propto P^{0}$, as suggested by Fig. 3 .

The observed friction on polyvinyl alcohol (PVA) gels sliding on a glass substrate is very near the theoretical one in the attractive case with a parameter $\delta \phi^{-1 / 2}=0.1$ (Fig. 5). ${ }^{1}$ In addition, the load dependence of friction of PVA gel is $f$ $\propto P^{0.2}$, which is quite near the predicted relations. Therefore, the interaction between PVA and the glass surface is suggested to be attractive with an attraction parameter $\delta \phi^{-1 / 2}$ of about 0.1 .

In this model, we have supposed that the interface between the gel and the solid has a contact in the macromolecular level. This assumption was justified by the experimental fact that highly water-swollen gels showed a linear dependence of the friction on the apparent contact area under

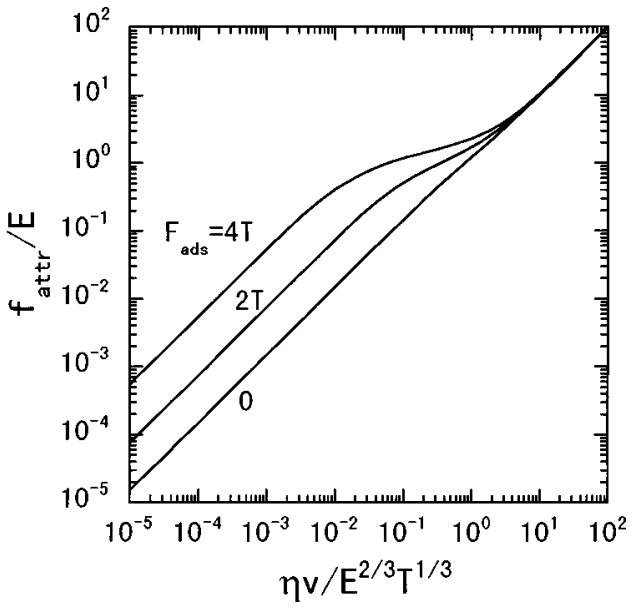

FIG. 6. Relation between the frictional forces and the normalized velocity $\alpha=\eta \nu / E^{2 / 3} T^{1 / 3}$ for gels sliding on attractive substrates with various $\delta \phi^{-1 / 2}$ values. $P=0$.

a wide pressure range. ${ }^{9}$ The nature of contact between a highly water-swollen gel and a solid is quite different than that between two solids. Since the elastic modulus of a gel usually lies in a range of $10^{3}-10^{6} \mathrm{~Pa}$, which is much smaller than that of a solid, a small pressure would be sufficient to cause the gel deforming to a molecular level contact with the opposing surface. In addition, surface tension of a gel also helps to make a molecular contact of gel with the solid surface. However, since a gel has a finite elastic modulus, the assumption of molecular level contact will only be valid when the pressure $P$ is moderately large in a scale of elastic modulus $E$ of the gel. Therefore, we might not be able to observe the limit $f \propto P^{0}$ when $P \rightarrow 0$ as shown in Fig. 4, in an experiment of macroscopic contact size, such as in an experiment using a tribometer. The gel friction might largely depend on the load even when the load is very small in terms of the elastic modulus of the gel.

As has been demonstrated by Eq. (44), the friction on a repulsive substrate has a linear dependence on the velocity. For the attractive substrate, the adsorption force increases first, then, via a maximum value, it decreases with the velocity, while the viscous friction increases with the velocity monotonously. Accordingly, the total friction of the gel will exhibit a large dependence on the velocity. Figure 6 shows the theoretical curves between the friction and the sliding velocity for attractive substrates of various $\delta \phi^{-1 / 2}$ values. The pressure is set to zero for simplicity. For weak adsorption, the viscous friction and the adsorbing friction are almost the same, so the gel only shows a monotonous increase with the velocity. For strong adsorption, the friction is mainly attributed to the adsorption when the velocity is low. After the maximum adsorption friction, the viscous friction becomes predominant.

As shown in the previous paper, for all gel samples measured in a velocity range of $\nu=10^{-4}-10^{-2} \mathrm{~m} / \mathrm{s}$, the frictional force increases with an increase in the sliding velocity. ${ }^{1}$ The double-logarithmic plots of $f$ against $\nu$ showed approximately linear relations in this velocity range. The slope of the lines changes from 0.21 to 0.67 , depending on the gel species as well as the water content of the gel. In the 
velocity range $\nu=10^{-4}-10^{-2} \mathrm{~m} / \mathrm{s}$, the normalized velocity parameter $\alpha=\nu \eta / E^{2 / 3} T^{1 / 3} \approx 10^{-4}-10^{-2}$ if we use $\eta$ $\approx 10^{-3} \mathrm{Ns} / \mathrm{m}^{2}$, and $E \approx 10^{5} \mathrm{~Pa}$. In this $\alpha$ range, the theoretical curve showed a linear relation between $f$ and $\alpha$ (or $\nu$ ) (Fig. 6) which does not perfectly agree with the observation. One reason for this discrepancy is probably due to the water loss of the sample during the prolonged measurement in air. Further experimental investigation in water is under progress.

The viscous friction of the solvent might be overestimated since we have used the Newtonian flow model with a no-slip boundary which is chosen to be located at a depth of $\sim R_{F}$ from the gel surface. The no-slip boundary might be much deeper than $R_{F}$, or a slip boundary should be taken into consideration. Otherwise, even at a very low sliding velocity, this thin shearing layer would give out a very strong shear strain rate (strain rate $=$ velocity/solvent layer thickness). For example, if we use parameters $\phi=0.04, a=3$ $\times 10^{-10} \mathrm{~m}$ for the gel, $R_{F}=3.4 \times 10^{-9} \mathrm{~m}$. When $\nu$ $=10^{-4} \mathrm{~m} / \mathrm{s}$, the strain rate $\sim 10^{5} \mathrm{~s}^{-1}$, which is very high and the flow is not Newtonian. ${ }^{10}$

In calculating the viscose force, we have simply used the viscosity for bulk water. Apparently, the viscosity of the solvent near the gel-solid interface would be quite different from that in the bulk state due to its strong interaction with the polymer. Therefore, $\eta$ might have complicated dependencies on $\nu, P, \phi$, and on the chemical structure of the gel. Besides, the behavior of a liquid trapped in a gap as narrow as several $\mathrm{nm}$ is expected to be much different than that of bulk liquid. ${ }^{11}$ Molecular triobology analysis of fluids has provided an experimental picture which shows that when the film is sufficiently thick (micrometers or more), it obeys continuum relations. At smaller film thickness (on the order of angstroms or nanometers), the films become solidlike in the sense that sliding does not occur unless a certain shear stress (or "yield stress") is attained. A detailed investigation of this is beyond the scope of this paper.
Although this model has ignored the numerical coefficients in the basic scaling relations and treated a cross-linked chain as a free one, the predictions of the frictional force on the dimensional factors of parameters $P, \nu, \phi, E, \delta$ have a good correspondence with the observations, which confirms the essential feature of the model.

This work may be extended to the case of gel-gel friction and find applications in understanding triobological behavior in biosystems or in development of biotechnology. ${ }^{12,13}$

\section{ACKNOWLEDGMENTS}

The authors express their sincere thanks to Y. Iwasaki and M. Higa for their experimental work. The authors also express their sincere thanks to Dr. S. A. Shaya (Johnson \& Johnson) for his valuable suggestions. This research was supported by Grant-in-aid for the Specially Promoted Research Project "Construction of Biomimetic Moving System Using Polymer Gels" from the Ministry of Education, Science and Culture, Japan.

${ }^{1}$ J. P. Gong, M. Higa, Y. Iwasaki, Y. Kastuyama, and Y. Osada, J. Phys. Chem. 101, 5487 (1997).

${ }^{2}$ C. W. McCutchen, Lubrication of Joints, The Joints and Synovial Fluid (Academic, New York, 1978), pp. 10, 437.

${ }^{3}$ M. Amontons, Mem. Acad. R. Sci. 1699, 206 (1699).

${ }^{4}$ A. W. Adamson, Physical Chemistry of Surfaces (Wiley, New York, 1990).

${ }^{5}$ P. G. de Gennes, Scaling Concept in Polymer Physics (Cornell University Press, Ithaca, NY, 1979).

${ }^{6}$ D. F. Moore, Principles and Applications of Tribology (Pergamon, Oxford, 1975).

${ }^{7}$ A. Shallamach, Wear 6, 375 (1968).

${ }^{8}$ G. Shah, P. L. Dubin, J. I. Kaplan, G. R. Newkome, C. N. Moorefield, and G. R. Baker, J. Colloid Interface Sci. 183, 397 (1996).

${ }^{9}$ J. P. Gong, Y. Iwasaki, G. Kagata, Y. Osada (in preparation).

${ }^{10}$ B. N. J. Persson, Sliding Friction: Physical Principles and Applications (Springer, New York, 1997).

${ }^{11}$ Fundamentals of Friction: Macroscopic and Microscopic Processes, edited by I. L. Singer and H. M. Pollock (Kluwer, Dordrect, 1992).

${ }^{12}$ J. C. Bray and E. W. Merrill, J. Biomed. Mater. Res. 7, 431 (1973).

${ }^{13}$ T. Sasada and K. Mabuchi, Proc. JSLE Int. Tribology Conf. 949 (1985). 